\title{
A New High-Intensity Synchrotron SIS100 with Strong Bunch Compression for GSI
}

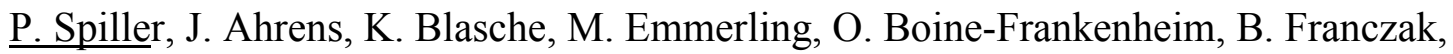 \\ P. Hülsmann, G. Moritz, C. Mühle, W.Vinzenz, G. Walter, P. Schütt \\ GSI, Darmstadt, Germany
}

\begin{abstract}
GSI has proposed a new synchrotron and storage ring facility for the future development of research in Nuclear Physics and in Plasma Physics. A new synchrotron accelerator $(\mathrm{BR}=100 \mathrm{Tm})$ with strong bunch compression capability will be the main machine in the new accelerator complex. One of its major design goals is to deliver intense uranium $(\mathrm{q}=28+$ ) beams at $0.4-2.7 \mathrm{GeV} / \mathrm{u}$ for the production and subsequent storage of exotic nuclei and antiprotons as well as for plasma physics experiments.

The $100 \mathrm{Tm}$ machine which is combined with a second synchrotron stage in the same machine tunnel $(B R=200$ $\mathrm{Tm}$ ) is equipped with a rf-compression system for the generation of short, high power heavy ion pulses. By means of this compression system single bunches of up to $20 \mathrm{~ns}$ can be generated in the fast extraction mode.
\end{abstract}

\section{DESIGN PARAMETERS}

The new synchrotron facility is designed for the acceleration of high intensity and high energy ion and proton beams. Two of the four proposed main research programs require compressed bunches of high intensity :

- For the radioactive beam program in storage rings about $1 \times 10^{12}$ uranium ions per second at energies from 400-1000 MeV/u in short bunches with pulse lengths from 50 to $100 \mathrm{~ns}$.

- For plasma physics research at least $1-2 \times 10^{12}$ uranium ions in a single short bunch (20-100 ns) at $400-2715 \mathrm{MeV} / \mathrm{u}$.

\section{CONCEPTUAL DESIGN}

The accelerator facility proposed for these research programs is based on the following accelerator stages :

I. The existing Unilac as injector for intermediate charged heavy ion beams.

II. The existing synchrotron (SIS12/18), which is operated as a fast-ramped booster synchrotron with up to four machine cycles per second (SIS12 mode).

III. A new large synchrotron SIS100, with fast-ramped superconducting magnets, designed for $\mathrm{BR}=100 \mathrm{Tm}, \mathrm{Bmax}=2 \mathrm{~T}$ and $\mathrm{dB} / \mathrm{dt}=4 \mathrm{~T} / \mathrm{s}$, which is used for the acceleration of heavy ions, e.g. U28+ions to $2715 \mathrm{MeV} / \mathrm{u}$.

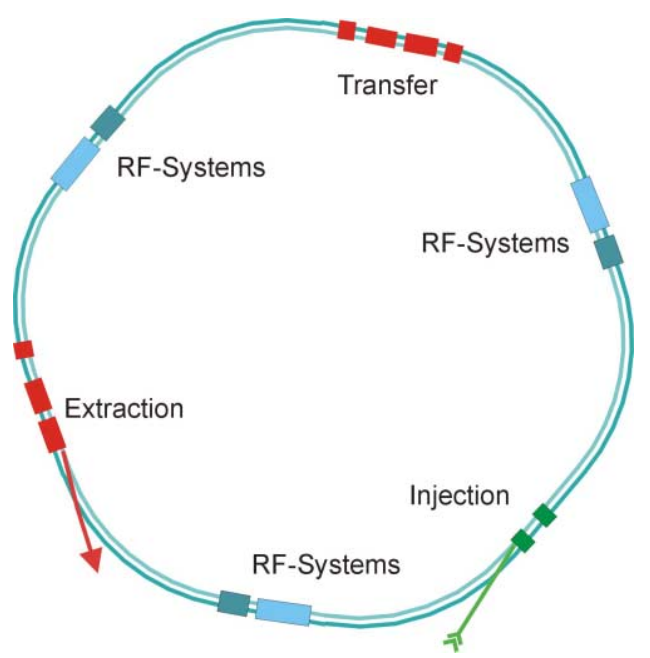

Fig. 1 : Layout of the two-stage synchrotron facility. Both rings have a circumference of $1083 \mathrm{~m}$ with six straight sections.

At present state of superconducting magnet technology it is economically not feasible to operate dipole magnets with $\cos \Theta$-coils at a ramp rate of $4 \mathrm{~T} / \mathrm{s}$. Therefore the first stage SIS100 with superconducting window-frame magnets based on the Nuklotron design was proposed [1].

The second stage SIS200 is used for the acceleration of heavy ions up to an energy of $23 \mathrm{GeV} / \mathrm{u}$ and can also serve as a stretcher ring to provide the required duty cycle of $100 \%$ for experiments with radioactive beams at the planned Super Fragment Separator (SFRS).

\section{GENERATION OF HIGH INTENSITY HEAVY ION BEAMS}

High intensity heavy ion beams are achieved by the acceleration of intermediate charge-state ions like U28+. Table 1 summarizes the design beam intensities for the accelerator chain from the Unilac to the SIS100 leading to an average beam intensity of $1 \times 10^{\mathbf{1 2}} \mathrm{U} 28+$-ions per second or $2 \times 10^{12} \mathrm{U}^{28+-}$ ions per pulse.

\begin{tabular}{llll}
\hline UNILAC & $\mathrm{U}^{4+}$ & $20 \mathrm{emA}$ & $11.4 \mathrm{MeV} / \mathrm{u}$ \\
\hline SIS12/18 & $\mathrm{U}^{28+}$ & $2.7 \times 10^{12} /$ cycle & $92 / 196 \mathrm{MeV} / \mathrm{u}$ \\
SIS100 & $\mathrm{U}^{28+}$ & $\begin{array}{l}1 \times 10^{12} / \text { sec. } / \\
2 \times 10^{12} / \text { pulse }\end{array}$ & $<1 \mathrm{GeV} / \mathrm{u}$ \\
& & & $0.2 .7 \mathrm{GeV} / \mathrm{u}$ \\
\hline
\end{tabular}

Table 1 : Intensities and number of particles along the accelerator chain. 
The existing SIS18 serves as a booster synchrotron, which can be run in a fast mode with four cycles per second at the reduced bending strength of $\mathrm{BR}=12 \mathrm{Tm}$. At the incoherent space-charge limit about $2.7 \times 10^{11} \mathrm{U}^{28+}$-ions are accelerated to $96 \mathrm{MeV} / \mathrm{u}$ in each cycle. By accumulation of four SIS12 batches the SIS100 will reach the design beam intensity of $1 \times 10^{12} \mathrm{U}^{28+}$-ions in a machine cycle of about 1 second (Fig. 2 ).

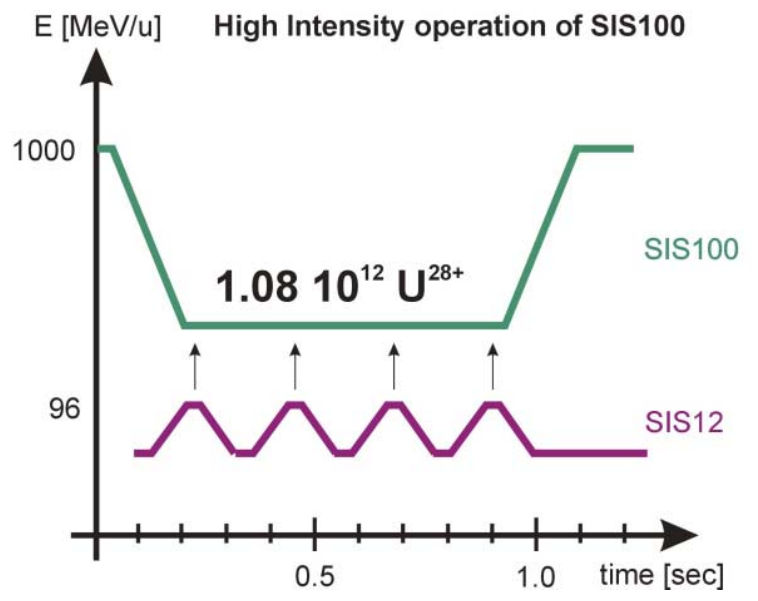

Fig. 2 : Machine cycles of the SIS100 and the SIS12/18 running in a fast ramped booster mode.

\section{LATTICE CONCEPT}

The preliminary layout of the SIS100 magnet lattice was determined by the following design features:

- six straight sections for beam injection, extraction and transfer from SIS100 to SIS200 and also for the two main RF-systems used for fast acceleration and for bunch compression

- triplet focusing in the SIS100 ring to provide large transverse acceptances with moderate magnet apertures

- missing magnet periods between the arcs and the straight sections to achieve zero dispersion in the straight sections.

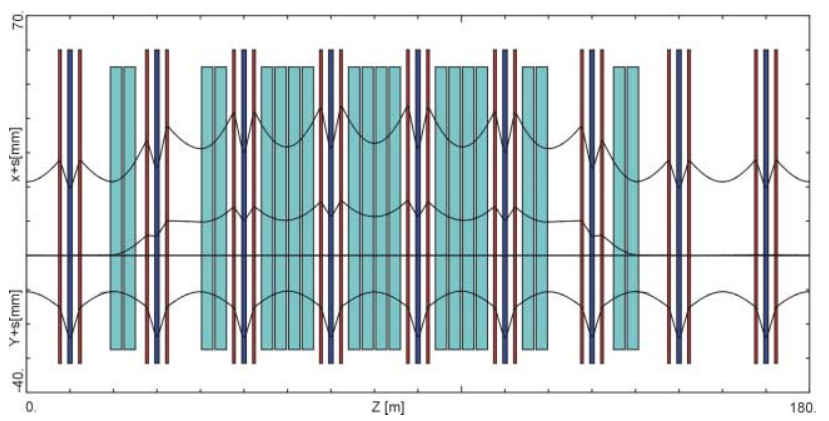

Figure 3 : Beam envelopes $\left(\varepsilon_{\mathrm{H}}=50 \mathrm{~mm} \mathrm{mrad}, \varepsilon_{\mathrm{V}}=15 \mathrm{~mm}\right.$ $\mathrm{mrad})$ and dispersion $(\delta \mathrm{p} / \mathrm{p}=0,01)$ in one superperiod of SIS 100

In Fig. 3 the beam envelopes and the dispersion function are plotted for one superperiod of the SIS100. Table 2 summarizes the present state of the SIS100 lattice parameters. The dipole magnets are designed with a rectangular aperture of $110 \mathrm{~mm} \times 55 \mathrm{~mm}$ and the quadrupoles with an aperture of $120 \times 63 \mathrm{~mm}$.

\begin{tabular}{ll}
\hline & \multicolumn{1}{c}{ SIS 100 } \\
\hline Number of Superperiods & 6 \\
Number of Periods & 54 triplett \\
Dipole Magnets: & 120 \\
$\quad$ Magnetic Field & $2 \mathrm{~T}$ \\
$\quad$ Straight Length & $2.618 \mathrm{~m}$ \\
$\quad$ Aperture & $110 \mathrm{~mm}$ x $55 \mathrm{~mm}$ \\
Quadrupole Magnets & 162 \\
$\quad$ Lengths [m] & $0.6 / 1.0 / 0.6$ \\
$\quad$ Quadrupole Strengths & $-36.5 /+38.3 /-36.5$ \\
$\quad$ & \\
$\quad$ Aperture & $120 \mathrm{~mm} \times 63 \mathrm{~mm}$ \\
Max. Beta Functions & $14.9 \mathrm{~m} / 37.0 \mathrm{~m}$ \\
Max. Dispersion & $1.4 \mathrm{~m}$ \\
Tunes & $15.9 / 15.7$ \\
\hline
\end{tabular}

Table 2 : Lattice Parameters of the SIS100.

\section{SINGLE BUNCH GENERATION AND COMPRESSION}

The single bunch structure is in general the nominal time structure at fast extraction. After acceleration, a single bunch is created by means of a dedicated rf-system which generates a dynamic barrier bucket. The bunch structure $(\mathrm{h}=20)$ after acceleration will be adiabaticly debunched within the barrier bucket and pre-compressed to a sufficiantly short single bunch. The barrier bucket rfsystem consists of low quality factor, broad band MAcavities.

However, high energy density in matter experiments [2] as well as an efficient radioactive beam production and storage [3], require further compression of the created single bunch.

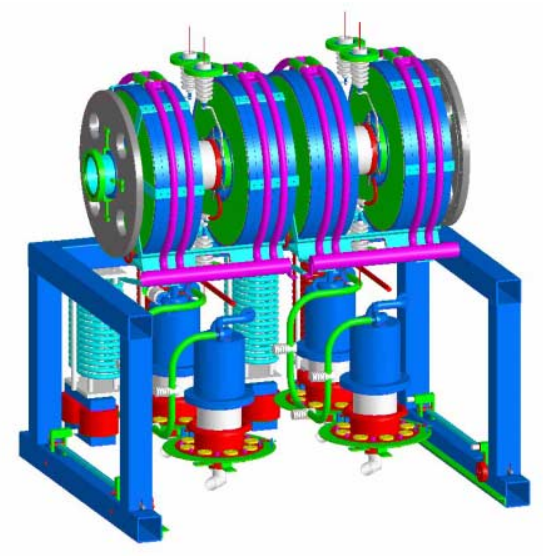

Figure 4 : Construction drawing of the prototype bunch compressor cavity for the SIS18 
A pulse length of $50 \mathrm{~ns}$ is calculated to match the hydrodynamic response of the fragmentation- and plasma physics target at an energy deposition of $50 \mathrm{~kJ} / \mathrm{g}$. This means a further compression of the pre-bunched beam by by a factor of 25 has to be achieved. The most economic concept for strong compression is the rf-voltage jump induced phase space rotation. A dedicated rf-system has to generate a sufficiantly high rf-voltage with an extremly short rise time - the full amplitude has to be reached within a time much shorter than the synchrotron period.

A prototyp of an appropriate MA-loaded compressor cavity and power amplifier is presently under development for the SIS18 (Figure 4) [4].

For fast compression of $\mathrm{U}^{28+}$ bunches in the SIS100 a total rf-voltage of $1.3 \mathrm{MV}$ is needed. The compressor system is operated at $\mathrm{h}=2$ which is still a rather low rffrequency of $465 \mathrm{kHz}$. Assuming a typical beam energy for RIB production of $1 \mathrm{GeV} / \mathrm{u}$, the compression process takes place over 110 revolutions. For the compression of proton beams at an energy of $29 \mathrm{GeV}$ only $500 \mathrm{kV}$ rfvoltage is required.

\begin{tabular}{|c|c|c|c|c|c|c|}
\hline & $\begin{array}{l}\text { Energy } \\
{[\mathrm{GeV} / \mathrm{u}]}\end{array}$ & $\begin{array}{l}\mathrm{h} \\
\#\end{array}$ & $\begin{array}{l}\text { RF- } \\
\text { Voltage } \\
{[\mathrm{MV}]}\end{array}$ & $\begin{array}{l}\text { Turns } \\
\#\end{array}$ & $\begin{array}{l}\text { Pulse } \\
\text { Length } \\
\text { [ns] }\end{array}$ & $\begin{array}{l}\mathrm{dp} / \mathrm{p} \\
{[\%]}\end{array}$ \\
\hline U28+ & 1 & 2 & 1.3 & 103 & 50 & 1 \\
\hline $\mathrm{p}$ & 29 & 2 & 0.5 & 510 & 5 & 1 \\
\hline
\end{tabular}

Table 3 : Compression data for the SIS100

At a design gap voltage of $40 \mathrm{kV}, 38$ compressor cavities of the SIS18-type are required (Table 4). Set-up as double cavity systems, nineteen modules will be installed in three of the six straight sections of the ring.

\begin{tabular}{ll}
\hline Number of compressor cavities & $32(2 \times 19)$ \\
\hline Operation frequency f [kHz] & $465 \pm 70$ \\
Harmonic number h & 2 \\
Gap voltage per cavity $\mathrm{V}_{0}[\mathrm{kV}]$ & 40 \\
Length of a double cavity [m] & 1.6 \\
Total rf-system length [m] & ca. $30 \mathrm{~m}$ \\
Total compression voltage [MV] & 1.3 \\
Total shunt impedance - cavities off $[\mathrm{k} \Omega]$ & 20 \\
Total shunt impedance - cavities on $[\mathrm{k} \Omega]$ & 51 \\
\hline
\end{tabular}

Table 4 : SIS100 MA-Compressor system

The compressor cavities will be inductively coupled to the power amplifier and driven by a push-pull operation of two Siemens tetrodes. For the generation of the full gap voltage, the working point of the tetrodes is moved to the so called A-mode. The shunt impedance per gap drops from $1.6 \mathrm{k} \Omega$ before operation to about $0.6 \mathrm{k} \Omega$ at maximum rf voltage.

\section{IMPEDANCE AND TUNE SHIFT}

It is essential to protect the bunch from interaction with the high total shunt impedance of the compressor and accelerator systems. Especially during the phase of prebunching where the shunt-impedances are much higher than during compression. One way to avoid the impedance coupling, is to short cicuit the cavity gap. However, mechanical relais would not be appropriated since the opening time must be very short $(<100 \mu \mathrm{s})$. Therefore we are presently testing high power MOSFET switches for shortening of the rf-gaps. The switches must resist at least the full gap voltage of $40 \mathrm{kV}$ if opened or on the other side an image current of $5 \mathrm{~A}$ if closed. At present we are testing a MOSFET-switch made by BEHLKE electronics. The switch has been installed at the SIS test cavity and succesfully operated in a c.W operation up to a rf-voltage of $10 \mathrm{kV}$, a current of $5 \mathrm{~A}$ and a frequency of $1 \mathrm{MHz}$.

Due to the compression the momentum spread rises up to $1 \%$ and causes a growth of the horizontal beam radius in the arcs of up to $1.4 \mathrm{~cm}$ (Table 2).

As a consequence of the increasing beam current a strong growth of the incoherent tune shift is expected. At a peak current of $\mathrm{I}_{\max }=130 \mathrm{~A}$ and an initial transverse emittance of $10 \times 10 \mathrm{~mm} \mathrm{mrad}$, the tune shift will be enhanced up to $\mathrm{dQ}=-1.2$. First results of particle tracking calculations indicate that unavoidable crossings of systematic resonances, may lead to a transverse emittance growth of a factor of two.

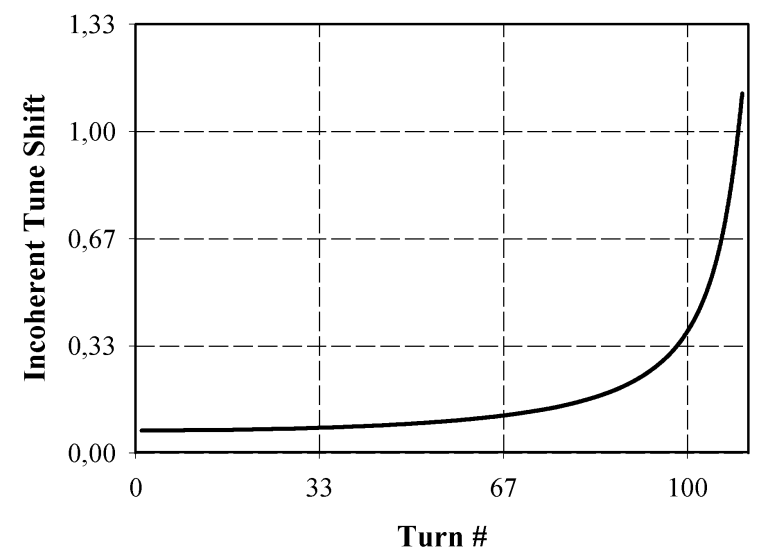

Figure 5 : Incoherent tune shift during compression of a $\mathrm{U}^{28}$-ion beam with an emittance of $10 \times 10 \mathrm{~mm}$ mrad.

During the next year, detailed theoretical studies on beam dynamics will be performed for the SIS100. A key issue for preparing the SIS100 compressor system is the set-up of the SIS18 compressor cavities in 2003.

\section{REFERENCES}

[1]G.Moritz, this conference

[2]N.A.Tahir et.al,Physics of Plasmas, Vol.7,No11(2000)

[3]N.A.Tahir et.al.,Phys. Rev. E, Vol. 63,036407-1(2001)

[4]K.Blasche et.al.,Proc. of the 6th EPAC-98, Stockholm 